\title{
Role of IL-17 gene polymorphisms in the susceptibility to gastric cancer
}

\author{
W.T. Qi', J.L. Gao ${ }^{2}$ and S.S. Zhang ${ }^{1}$ \\ ${ }^{1}$ Department of Emergency, Zhengzhou People's Hospital, Zhengzhou, China \\ 2Department of Internal Medicine, Zhengzhou Straight Authority Hospital, \\ Zhengzhou, China
}

Corresponding author: W.T. Qi

E-mail: qi_wentao2@163.com

Genet. Mol. Res. 14 (4): 13364-13369 (2015)

Received April 16, 2015

Accepted June 17, 2015

Published October 26, 2015

DOI http://dx.doi.org/10.4238/2015.October.26.33

ABSTRACT. We conducted a study to investigate the role of three IL-17 gene single nucleotide polymorphisms (SNP) (rs2275913G>A, rs3748067C $>\mathrm{T}$, and $\mathrm{rs} 763780 \mathrm{~T}>\mathrm{C}$ ) in the development of gastric cancer. A total of 252 patients with gastric cancer and 252 control subjects were collected between May 2012 and May 2014. The SNP genotyping of $I L-17 A$ rs2275913G $>A$ and $r s 3748067 C>T$ and $I L-17 F$ rs763780 T>C was performed using the Sequenom MassARRAY platform (Sequenom, San Diego, CA, USA) according to the manufacturer instructions. By conditional regression analysis, individuals carrying the $A A$ and the GA+AA genotypes of rs2275913G>A were correlated with an elevated risk of gastric cancer when compared with those carrying the GG genotype, and the adjusted ORs (95\%Cls) were 2.05 (1.13-3.76) for the AA genotype and 1.45 (1.03-2.08) for the GA+AA genotype. In conclusion, our results suggest that the $I L-17 A$ rs3748067C $>T$ and $I L$ $17 F$ rs763780 $\mathrm{T}>\mathrm{C}$ polymorphisms play an important role in the risk of gastric cancer in a Chinese population.

Key words: IL-17; Polymorphism; Gastric cancer; Chinese population 


\section{INTRODUCTION}

Gastric cancer (GC) is the fourth most common cancer in the world and the second most common cause of cancer-related death (International Agency for Research on Cancer, 2012). Although genetic and environmental factors in addition to Helicobacter pylori infections have been identified as playing important roles in the development of GC (Houghton and Wang, 2005; Merchant, 2005; Dong et al., 2008), the precise etiology of the disease remains unclear.

Chronic inflammation is a well-known risk factor for malignant transformation, but the role inflammation plays in cancer initiation is poorly understood (Scheller et al., 2006; Candido and Hagemann, 2013). Interleukin-17 (IL-17) is a novel cytokine family that consists of six protein members (IL-17A-F) that play important roles in many chronic inflammatory diseases and in cancer development (Wu et al., 2013). Several previous studies have reported that the over-expression of IL-17A and IL-17F could be found in various tumor tissues such as breast cancer, GC, and nonsmall cell lung cancer (Meng et al., 2012; Yang et al., 2012; Cheng et al., 2015). Single nucleotide polymorphisms (SNPs) are an important type of gene mutation that can influence the transcription and translation of the associated gene. Previous studies have reported an association between IL-17 gene polymorphisms and the risk of GC, but the results have been inconsistent (Qinghai et al., 2014; Wang et al., 2014; Yu et al., 2014; Gao et al., 2015). We therefore conducted a study to investigate the role of 3 SNPs in $I L-17$ (rs2275913G >A, rs3748067C > T, and rs763780 T>C) in the development of GC.

\section{MATERIAL AND METHODS}

\section{Patients}

A total of 252 patients with GC and 252 control subjects were collected from Zhengzhou People's Hospital between May 2012 and May 2014. All patients with GC were histopathologically confirmed. The exclusion criteria were the presence of any other malignant tumors or inadequate organ function. To obtain frequency-matched controls on age and gender, 252 control subjects were randomly selected from the Health Check-up Center of the Zhengzhou People's Hospital during the same period. Control subjects who had a history of cancer were excluded from our study.

Demographic and related lifestyle factors of patients with GC and control subjects were collected using a face-to-face questionnaire and included gender, age, dietary habits, alcohol consumption, tobacco smoking, and family history of cancer.

Enzyme-linked immunoabsorbent assay (ELISA) (Diagnostic Automation, USA) and a rapid urea breath test were performed to assess the $H$. pylori infection state of patients with GC and controls.

Informed consent was obtained from all patients and control subjects or their relatives before enrollment in this study. The study protocol was approved by the Ethics Committee of Zhengzhou People's Hospital.

\section{Genotyping}

Each patient and control subject was asked to provide $5 \mathrm{~mL}$ venous blood for 
genomic DNA extraction. The blood samples were kept at $-20^{\circ} \mathrm{C}$ until use, with $0.5 \mathrm{mg} / \mathrm{mL}$ ethylenediaminetetraacetic acid used as an anticoagulant. Samples were placed randomly onto 384well plates and were blinded for disease status. The design of the assay and the SNP genotyping of IL-17A rs2275913G >A and rs3748067C > T and IL-17F rs763780 T>C were performed by Bio Miao Biological Technology (Beijing, China) using the Sequenom MassARRAY platform (Sequenom, San Diego, CA, USA) according to the manufacturer instructions. The results of all duplicated samples were $100 \%$ consistent.

\section{Statistical analysis}

The chi-square $\left(\mathrm{X}^{2}\right)$ test was used to compare the differences in gender, age, dietary habits, alcohol consumption, tobacco smoking, and family history of cancer, and also the frequency distributions of genotypes and alleles between patients and controls. A $\mathrm{X}^{2}$ was taken to evaluate the Hardy-Weinberg equilibrium (HWE) of the control groups. Unconditional logistic regression analysis was performed to calculate ORs and $95 \% \mathrm{Cls}$ as estimates of the relative risk for each SNP, with adjustment for potential confounding factors. The most common genotype in the controls was used as the reference group. All $P$ values were two-sided, and a $P$ value $<0.05$ was considered to be statistically significant. The SPSS 16.0 (SPSS, Inc, Chicago, IL, USA) software was used for statistical analyses.

\section{RESULTS}

Demographic and clinical characteristics of patients with GC and control subjects are summarized in Table 1. The mean ages of patients with GC and control subjects were $57.72 \pm$ 10.75 and $58.25 \pm 11.25$ years, respectively. As expected, there were no significant differences in gender and age between patients and control subjects $(P>0.05)$. We found that patients with $G C$ were more likely to be alcohol drinkers, have cancer history in the first relatives, and be infected with H. pylori.

Table 1. Demographic and clinical characteristics of patients with gastric cancer and control subjects.

\begin{tabular}{|c|c|c|c|c|c|c|}
\hline & Patients $(N=252)$ & $\%$ & Controls $(N=252)$ & $\%$ & $t$ or $x^{2}$ & $P$ value \\
\hline Age (mean $\pm S D$, years) & $57.72 \pm 10.75$ & 54.37 & $58.25 \pm 11.25$ & & 0.54 & 0.29 \\
\hline$<60$ & 137 & & 133 & 52.78 & & \\
\hline$\geq 60$ & 115 & 45.63 & 119 & 47.22 & 0.13 & 0.72 \\
\hline \multicolumn{7}{|l|}{ Gender } \\
\hline Female & 97 & 38.49 & 97 & 38.49 & & \\
\hline Male & 155 & 61.51 & 155 & 61.51 & 0.00 & 1.00 \\
\hline \multicolumn{7}{|c|}{ Cancer history in first degree relatives } \\
\hline No & 236 & 93.65 & 251 & 99.60 & & \\
\hline Yes & 16 & 6.35 & 1 & 0.40 & 13.70 & $<0.001$ \\
\hline \multicolumn{7}{|l|}{ Alcohol drinking [N (\%)] } \\
\hline Never & 134 & 53.17 & 162 & 64.29 & & \\
\hline Current or ever & 118 & 46.83 & 90 & 35.71 & 6.42 & 0.01 \\
\hline \multicolumn{7}{|l|}{ Tobacco smoking [N (\%)] } \\
\hline Never & 161 & 63.89 & 172 & 68.25 & & \\
\hline Current or ever & 91 & 36.11 & 80 & 31.75 & 1.07 & 0.30 \\
\hline \multicolumn{7}{|l|}{ Helicobacter pylori } \\
\hline Negative & 113 & 44.84 & 198 & 78.57 & & \\
\hline Positive & 139 & 55.16 & 54 & 21.43 & 60.67 & $<0.001$ \\
\hline
\end{tabular}


In our study population, the genotype and allele distributions of IL-17A rs2275913G>A conformed to HWE in the control group, while those of $I L-17 A$ rs3748067C $>T$ and $I L-17 F$ rs763780 $\mathrm{T}>\mathrm{C}$ did not (Table 2). The minor allele frequencies of $I L-17 A$ rs2275913G $>A$, rs3748067C $>T$, and IL17F rs763780 T>C were similar to those in the dbSNP database (http://www.ncbi.nlm.nih.gov/SNP/).

By conditional regression analysis, individuals carrying the $A A$ genotype and the GA+AA genotype of IL-17 rs2275913G>A were correlated with an elevated risk of GC when compared with those carrying the GG genotype, and the adjusted ORs (95\%Cls) were 2.05 (1.13-3.76) for the AA genotype and $1.45(1.03-2.08)$ for the GA+AA genotype (Table 3$)$. However, the IL$17 A$ rs3748067C $>T$ and $I L-17 F$ rs763780T $>C$ gene polymorphisms were not associated with an increased risk of GC.

\begin{tabular}{|c|c|c|c|c|c|c|c|}
\hline \multirow[t]{2}{*}{ IL-17 genotype } & \multirow[t]{2}{*}{ Patients } & \multirow[t]{2}{*}{$\%$} & \multirow[t]{2}{*}{ Controls } & \multirow[t]{2}{*}{$\%$} & \multirow[t]{2}{*}{ HWE } & \multicolumn{2}{|c|}{ Minor allele frequency (MAF) } \\
\hline & & & & & & In controls & In database \\
\hline \multicolumn{8}{|l|}{ rs2275913G>A } \\
\hline GG & 100 & 39.68 & 122 & 48.41 & & & \multirow{4}{*}{0.2927} \\
\hline GA & 110 & 43.65 & 105 & 41.67 & \multirow{3}{*}{0.47} & \multirow{3}{*}{0.3075} & \\
\hline AA & 42 & 16.67 & 25 & 9.92 & & & \\
\hline $\mathrm{GA}+\mathrm{AA}$ & 152 & 60.32 & 130 & 51.59 & & & \\
\hline \multicolumn{8}{|l|}{ rs3748067C>T } \\
\hline $\mathrm{TT}$ & 211 & 83.73 & 221 & 87.70 & & \multirow{4}{*}{0.0794} & \multirow{4}{*}{0.0769} \\
\hline TC & 25 & 9.92 & 22 & 8.73 & \multirow{3}{*}{$<0.05$} & & \\
\hline $\mathrm{CC}$ & 16 & 6.35 & 9 & 3.57 & & & \\
\hline $\mathrm{TC}+\mathrm{CC}$ & 41 & 16.27 & 31 & 12.30 & & & \\
\hline \multicolumn{8}{|l|}{ rs763780T $>C$} \\
\hline $\mathrm{CC}$ & 203 & 80.56 & 213 & 84.52 & \multirow{4}{*}{$<0.05$} & \multirow{4}{*}{0.1032} & \multirow{4}{*}{0.0935} \\
\hline СT & 29 & 11.51 & 26 & 10.32 & & & \\
\hline TT & 20 & 7.94 & 13 & 5.16 & & & \\
\hline $\mathrm{CT}+\mathrm{TT}$ & 49 & 19.44 & 39 & 15.48 & & & \\
\hline
\end{tabular}

HWE = Hardy-Weinberg equilibrium.

\begin{tabular}{|c|c|c|c|c|c|c|}
\hline IL-17 genotype & Patients & $\%$ & Controls & $\%$ & OR $(95 \% \mathrm{Cl})^{1}$ & $P$ value \\
\hline \multicolumn{7}{|l|}{ rs2275913G $>A$} \\
\hline GG & 100 & 39.68 & 122 & 48.41 & 1.0 (Ref.) & - \\
\hline GA & 110 & 43.65 & 105 & 41.67 & $1.28(0.86-1.89)$ & 0.20 \\
\hline$A A$ & 42 & 16.67 & 25 & 9.92 & $2.05(1.13-3.76)$ & 0.01 \\
\hline $\mathrm{GA}+\mathrm{AA}$ & 152 & 60.32 & 130 & 51.59 & $1.45(1.03-2.08)$ & 0.04 \\
\hline \multicolumn{7}{|l|}{ rs3748067C>T } \\
\hline TT & 211 & 83.73 & 221 & 87.70 & 1.0 (Ref.) & - \\
\hline TC & 25 & 9.92 & 22 & 8.73 & $1.19(0.62-2.29)$ & 0.57 \\
\hline $\mathrm{CC}$ & 16 & 6.35 & 9 & 3.57 & $1.86(0.75-4.88)$ & 0.14 \\
\hline $\mathrm{TC}+\mathrm{CC}$ & 41 & 16.27 & 31 & 12.30 & $1.39(0.81-2.37)$ & 0.20 \\
\hline \multicolumn{7}{|l|}{ rs763780T $>C$} \\
\hline $\mathrm{CC}$ & 203 & 80.56 & 213 & 84.52 & 1.0 (Ref.) & - \\
\hline $\mathrm{CT}$ & 29 & 11.51 & 26 & 10.32 & $1.17(0.64-2.15)$ & 0.58 \\
\hline TT & 20 & 7.94 & 13 & 5.16 & $1.61(0.74-3.63)$ & 0.19 \\
\hline $\mathrm{CT}+\mathrm{TT}$ & 49 & 19.44 & 39 & 15.48 & $1.32(0.81-2.15)$ & 0.24 \\
\hline
\end{tabular}

${ }_{1}$ Adjusted for gender, age, cancer history in first degree relatives, alcohol drinking, and Helicobacter pylori status.

\section{DISCUSSION}

It is well known that IL-17 is an important cytokine family containing six homologous 
members (IL-17A-F), which has an important function in connecting innate and adaptive immunities (Kolls and Lindén, 2004). Previous studies have shown that IL-17 is an essential proinflammatory cytokine that evokes the production of many additional cytokines and chemokine secretion through various cell types such as mesenchymal and myeloid cells, in order to recruit monocytes and neutrophils into the microenvironment of the site of inflammation (Iwakura et al., 2011). In addition, IL-17 plays important roles in promoting the expression of antimicrobial peptides and in facilitating host defense against infections (Kao et al., 2004; Matsuzaki and Umemura, 2007).

To date, many molecular epidemiological studies have evaluated the association between IL-17 gene polymorphisms and the development of GC, but the published results have been conflicting. For the association between IL-17A rs2275913G>A and the risk of GC, six studies reported different results (Shibata et al., 2009; Arisawa et al., 2012; Qinghai et al., 2014; Wang et al., 2014; Zhang et al., 2014; Gao et al., 2015). Shibata et al. (2009) and Arisawa et al. (2012) reported that the A allele of IL-17A rs2275913G>A was significantly associated with the development of GC in Japanese populations. In addition, Zhang et al. (2014) conducted a casecontrol study in a Chinese population and reported that the IL-17A rs2275913G>A polymorphism increased GC risk, and interacted with $H$. pylori infection. Qinghai et al. (2014) and Wang et al. (2014) also conducted case-control studies in a Chinese population and suggested that the $I L-17 \mathrm{~A}$ rs2275913G >A polymorphism increased the risk of GC. However, the case-control study of Gao et al. (2015), also in a Chinese population, did not find significant association between the IL-17A rs2275913G >A polymorphism and the risk of developing GC. In our study, we found that the AA and $G A+A A$ genotypes of $I L-17 A$ rs2275913G $>A$ were correlated with an elevated risk of GC. The discrepancies between these results might be caused by differences in ethnicities, study design, and sample size.

We identified three limitations in this study. First, patients and controls were selected from one hospital, and IL-17A rs3748067C $>T$ and $I L-17 F$ rs763780 T>C were not in accordance with HWE in the control group, which suggests that the sample used by this study would not likely be representative of other populations. Second, the sample size of patients with GC was relatively small, which might limit the statistical power to find an association between groups. Third, the risk of GC might be modified by many other cytokine genes in addition to the $I L-17$ genes. Therefore, further studies with larger sample sizes are greatly needed to assess the association between IL17 gene polymorphisms and the risk of GC.

In conclusion, our results suggest that the IL-17A rs3748067C>T and IL-17F rs763780 $\mathrm{T}>\mathrm{C}$ polymorphisms play an important role in the risk of $\mathrm{GC}$ in a Chinese population. Further studies involving various populations and large sample sizes are greatly needed to confirm our results.

\section{Conflicts of interest}

The authors declare no conflict of interest.

\section{REFERENCES}

\footnotetext{
Arisawa T, Tahara T, Shiroeda H, Matsue $\mathrm{Y}$, et al. (2012). Genetic polymorphisms of IL17A and pri-microRNA-938, targeting IL17A 3'-UTR, influence susceptibility to gastric cancer. Hum. Immunol. 73: 747-752.

Candido J and Hagemann T (2013). Cancer-related inflammation. J. Clin. Immunol. 33: S79-84.

Cheng S, Shao Z, Liu X, Guo L, et al. (2015). Interleukin 17A polymorphism elevates gene expression and is associated with increased risk of nonsmall cell lung cancer. DNA Cell Biol. 34: 63-68.
} 
Dong LM, Potter JD, White E, Ulrich CM, et al. (2008). Genetic susceptibility to cancer: the role of polymorphisms in candidate genes. JAMA 299: 2423-2436.

Gao YW, Xu M, Xu Y, Li D, et al. (2015). Effect of three common IL-17 single nucleotide polymorphisms on the risk of developing gastric cancer. Oncol. Lett. 9: 1398-1402.

Houghton J and Wang TC (2005). Helicobacter pylori and gastric cancer: a new paradigm for inflammation-associated epithelial cancers. Gastroenterology 128: 1567-1578.

International Agency for Research on Cancer (2012). Stomach cancer. estimated incidence, mortality and prevalence worldwide in 2012. In: Globocan 2012: Estimated cancer incidence, mortality and prevalence worldwide in 2012. World Health Organization. Available at [http://globocan.iarc.fr/Pages/fact_sheets_cancer.aspx].

Iwakura Y, Ishigame H, Saijo S and Nakae S (2011). Functional specialization of interleukin-17 family members. Immunity 34: 149-162.

Kao CY, Chen Y, Thai P, Wachi S, et al. (2004). IL-17 markedly up-regulates beta-defensin-2 expression in human airway epithelium via JAK and NF-kappaB signaling pathways. J. Immunol. 173: 3482-3491.

Kolls JK and Lindén A (2004). Interleukin-17 family members and inflammation. Immunity 21: 467-476.

Matsuzaki G and Umemura M (2007). Interleukin-17 as an effector molecule of innate and acquired immunity against infections. Microbiol. Immunol. 51: 1139-1147.

Meng XY, Zhou CH, Ma J, Jiang C, et al. (2012). Expression of interleukin-17 and its clinical significance in gastric cancer patients. Med. Oncol. 29: 3024-3028.

Merchant JL (2005). Inflammation, atrophy, gastric cancer: connecting the molecular dots. Gastroenterology 129: $1079-1082$.

Qinghai Z, Yanying W, Yunfang C, Xukui Z, et al. (2014). Effect of interleukin-17A and interleukin-17F gene polymorphisms on the risk of gastric cancer in a Chinese population. Gene 537: 328-332.

Scheller J, Ohnesorge N and Rose-John S (2006). Interleukin-6 trans-signalling in chronic inflammation and cancer. Scand. J. Immunol. 63: 321-329.

Shibata T, Tahara T, Hirata I and Arisawa T (2009). Genetic polymorphism of interleukin-17A and -17F genes in gastric carcinogenesis. Hum. Immunol. 70: 547-551.

Wang N, Yang J, Lu J, Qiao Q, et al. (2014). IL-17 gene polymorphism is associated with susceptibility to gastric cancer. Tumour Biol. 35: 10025-10030.

Wu D, Wu P, Huang Q, Liu Y, et al. (2013). Interleukin-17: A promoter in colorectal cancer progression. Clin. Dev. Immunol. 2013: 436307.

Yang L, Qi Y, Hu J, Tang L, et al. (2012). Expression of Th17 cells in breast cancer tissue and its association with clinical parameters. Cell. Biochem. Biophys. 62: 153-159.

Yu H, Sun S, Liu F and Xu QH (2014). Meta-analysis of associations between interleukin-17 gene polymorphisms and risk of gastric cancer. Asian Pac. J. Cancer Prev. 15: 8709-8713.

Zhang X, Zheng L, Sun Y and Zhang X (2014). Analysis of the association of interleukin-17 gene polymorphisms with gastric cancer risk and interaction with Helicobacter pylori infection in a Chinese population. Tumour Biol. 35: $1575-1580$. 